\title{
BMJ Open Application of speCtraL computed tomogrAphy to impRove specIficity of cardiac compuTed tomographY (CLARITY study): rationale and design
}

\author{
Robbert Willem van Hamersvelt, ${ }^{1}$ Ivana Išgum, ${ }^{2}$ Pim A de Jong, ${ }^{1}$ \\ Maarten Jan Maria Cramer, ${ }^{3}$ Geert E H Leenders, ${ }^{3}$ Martin J Willemink, ${ }^{1}$ \\ Michiel Voskuil, ${ }^{3}$ Tim Leiner $^{1}$
}

To cite: van Hamersvelt RW, Išgum I, de Jong PA, et al. Application of speCtraL computed tomogrAphy to impRove speclficity of cardiac compuTed tomographY (CLARITY study): rationale and design. BMJ Open 2019;9:e025793. doi:10.1136/ bmjopen-2018-025793

- Prepublication history for this paper is available online. To view these files, please visit the journal online (http://dx.doi org/10.1136/bmjopen-2018025793).

Received 1 August 2018 Revised 24 December 2018 Accepted 25 January 2019

Check for updates

(c) Author(s) (or their employer(s)) 2019. Re-use permitted under CC BY-NC. No commercial re-use. See rights and permissions. Published by BMJ.

${ }^{1}$ Department of Radiology, University Medical Centre Utrecht, Utrecht University, Utrecht, The Netherlands ${ }^{2}$ Image Sciences Institute, University Medical Centre Utrecht, Utrecht University, Utrecht, The Netherlands ${ }^{3}$ Department of Cardiology, University Medical Centre Utrecht, Utrecht University, Utrecht, The Netherlands

Correspondence to Robbert Willem van Hamersvelt; r.w.vanhamersvelt-3@ umcutrecht.nl

\section{ABSTRACT}

Introduction Anatomic stenosis evaluation on coronary CT angiography (CCTA) lacks specificity in indicating the functional significance of a stenosis. Recent developments in $\mathrm{CT}$ techniques (including dual-layer spectral detector CT [SDCT] and static stress CT perfusion [CTP]) and image analyses (including fractional flow reserve [FFR] derived from CCTA images [ $\mathrm{FFR}_{\mathrm{CT}}$ ] and deep learning analysis [DL]) are potential strategies to increase the specificity of CCTA by combining both anatomical and functional information in one investigation. The aim of the current study is to assess the diagnostic performance of (combinations of) SDCT, CTP, FFR ${ }_{\mathrm{CT}}$ and DL for the identification of functionally significant coronary artery stenosis.

Methods and analysis Seventy-five patients aged 18 years and older with stable angina and known coronary artery disease and scheduled to undergo clinically indicated invasive FFR will be enrolled. All subjects will undergo the following SDCT scans: coronary calcium scoring, static stress CTP, rest CCTA and if indicated (history of myocardial infarction) a delayed enhancement acquisition. Invasive FFR of $\leq 0.80$, measured within 30 days after the SDCT scans, will be used as reference to indicate a functionally significant stenosis. The primary study endpoint is the diagnostic performance of SDCT (including CTP) for the identification of functionally significant coronary artery stenosis. Secondary study endpoint is the diagnostic performance of SDCT, CTP, FFR and DL separately and combined for the identification of functionally significant coronary artery stenosis.

Ethics and dissemination Ethical approval was obtained. All subjects will provide written informed consent. Study findings will be disseminated through peer-reviewed conference presentations and journal publications. Trial registration number NCT03139006; Pre-results.

\section{INTRODUCTION}

Anatomic evaluation of luminal stenosis on coronary CT angiography (CCTA) has high diagnostic performance to detect and rule out coronary artery disease. ${ }^{1-3}$ However, it lacks specificity in indicating the extent of myocardial ischaemia caused by a stenosis, especially
Strengths and limitations of this study

Both anatomical and functional cardiac information will be evaluated a single CT investigation.

- A comprehensive set of CT techniques will be evaluated.

- Results of this study will clarify to what extent (combinations of) CT techniques can improve diagnostic performance of $\mathrm{CT}$.

- As a monocentre study, generalisation of the results may be limited.

- Given the high prevalence of disease in our population, a potential limitation is selection bias.

in the presence of calcified lesions, due to volume artefacts caused by blooming. ${ }^{1-3}$ Invasively measured fractional flow reserve (FFR) is currently used to identify functionally significant coronary artery lesions and to guide revascularisation treatment. ${ }^{4}$ Results of the ISCHEMIA trial (NCT01471522) are awaited to shed light on the ongoing discussion concerning outcomes with revascularisation based only on ischaemia. Until these results are published, cardiac CT is the single modality able to simultaneously evaluate coronary artery anatomy and functional information in one investigation non-invasively. Recent developments in CT acquisition and image analysis techniques, such as dual-energy CT (DECT), static stress CT perfusion imaging (CTP), FFR derived from CCTA images $\left(\mathrm{FFR}_{\mathrm{CT}}\right)$ and deep learning (DL) based image analysis, have shown the potential to improve the specificity of CCTA by combining both anatomical and functional information in one investigation. ${ }^{5-12}$

Dual-layer spectral detector CT (SDCT) is a DECT system with one X-ray tube and a dual-layer detector, which separately detects low and high-energy photons. In this way, 
there is no time delay between the acquisitions of the different energy levels resulting in temporally coregistered dual-energy data. Also, the acquired images are spatially coregistered, making this DECT technique ideal for imaging a moving heart. ${ }^{13}$ In addition, by combining the information from both detector layers a conventional image can be reconstructed. ${ }^{14}$ Virtual monoenergetic images (40-200 keV) reconstructed from the SDCT may reduce blooming and beam-hardening artefacts and increase in-stent lumen visibility. ${ }^{15}{ }^{16} \mathrm{~A}$ plaque-specific monoenergetic level can be applied to improve detection and quantification of coronary plaques. ${ }^{17}$ Together with the opportunity to assess myocardial iodine distribution and quantification in both rest and static stress images, ${ }^{9} 18$ SDCT may allow for improved anatomical and functional evaluation.

For $\mathrm{FFR}_{\mathrm{CT}}$, the coronary lumen is segmented on CCTA images and assessed by algorithms based on fluid dynamics, lumped parameter models or machine learning. ${ }^{56}$ In this way, $\mathrm{FFR}_{\mathrm{CT}}$ is able to evaluate the functional significance of stenosis and has shown to improve diagnostic performance of CT. ${ }^{56}$ With SDCT, a plaque-specific monoenergetic level can be applied to improve lumen segmentation and the performance of FFR $_{\mathrm{CT}}$.

$\mathrm{DL}$ is a machine learning-based approach that has been shown to outperform other image analysis approaches in a wide variety of medical image analysis tasks. ${ }^{19}$ One of these tasks includes segmentation and detection analysis of cardiac CT. ${ }^{11} 121920$ The growing amount of data generated by new CT acquisition techniques (eg, SDCT and CTP) makes DL analysis a technique of high interest. A recent study showed that patients with functionally significant coronary artery stenosis can be identified using DL analysis on CCTA. ${ }^{11} 12$ By combining these new technologies (SDCT, CTP, FFR ${ }_{\mathrm{CT}}$ and DL), we hypothesise more accurate information will be available about the coronary anatomy, degree of stenosis, FFR $_{\mathrm{CT}}$ and myocardial perfusion, thereby contributing to a higher specificity of CT for identification of functionally significant coronary artery stenosis. To test this hypothesis, we have designed the CLARITY study (Application of speCtraL computed tomogrAphy to impRove specIficity of cardiac compuTed tomographY). This paper describes the methods, rationale and design of the study.

\section{METHODS AND ANALYSIS \\ Study design}

The CLARITY study is a prospective monocentre diagnostic study designed to assess the accuracy of SDCT, CTP, FFR $\mathrm{CT}_{\mathrm{C}}$ and DL-based image analysis as a non-invasive way for the identification of functionally significant coronary artery stenosis, using invasive FFR as the standard of reference. Combinations of multiple new CT acquisition techniques (ie, SDCT and CTP) and image analysis techniques (ie, FFR ${ }_{\mathrm{CT}}$ and DL) will be investigated to evaluate the optimal diagnostic performance of SDCT for the identification of functionally significant coronary artery stenosis.

\section{Study objectives}

The primary study objective is to assess the diagnostic performance of SDCT (including CTP) for the identification of functionally significant coronary artery stenosis in patients with stable angina and known coronary artery disease, using invasive FFR as the standard of reference in a patient-based analysis. The secondary objective is to assess the diagnostic performance of SDCT, CTP, FFR $_{\mathrm{CT}}$ and DL separately and combined for the identification of functionally significant coronary artery stenosis in patients with stable angina and known coronary artery disease, using invasive FFR as the standard of reference in a vessel-based and patient-based analysis. The tertiary objective is to investigate if and to what extent SDCT can decrease the degree of blooming and beam-hardening artefacts of calcifications.

Patients will only be included in the study after informed consent is signed.

\section{Targeted population}

Patients aged 18 years and older with stable angina and known coronary artery disease and scheduled to undergo clinically indicated invasive FFR are eligible for inclusion. Further inclusion and exclusion criteria are described in table 1 . Due to the high negative predictive value of CCTA, patients are usually referred to CCTA when the pretest likelihood for obstructive coronary artery disease is $<50 \%{ }^{21}$ Because CCTA has a moderate specificity in indicating the functional significance of a stenosis, it is not (yet) recommended for patients with pretest likelihood higher than $50 \% .{ }^{21}$ Because we are interested in increasing the specificity of CCTA, we have chosen to include patients with stable angina and known coronary artery disease.

\section{Patient and public involvement}

Neither patients nor the public were involved in the design of the study, development of the research question and deciding on the outcome measures. At inclusion, patients are asked if they would like to receive results of the study at the group level. After completion of the study, results will be disseminated through a report to the patients who wish to receive information about the study.

\section{Sample size calculation}

To allow for evaluation of sensitivity and specificity within our study population, the proportions of cases $($ FFR $\leq 0.80)$ and controls (FFR $>0.8)$ in our sample should take the prevalence of disease into account. Internal research of patient records of the past 3 years showed that $50 \%$ of the patients who underwent invasive FFR had at least one positive FFR measurement $(\mathrm{FFR} \leq 0.80)$. With a prevalence of 0.5 , the proportion of cases to controls is 1 , indicating that the same amount of cases and controls is required. The main goal of the CLARITY study is to increase the specificity of SDCT (including CTP) for the 


\begin{tabular}{|c|c|}
\hline Inclusion criteria & Exclusion criteria \\
\hline $\begin{array}{l}\text { Age } \geq 18 \text { years } \\
\text { Stable angina and } \\
\text { known coronary } \\
\text { artery disease } \\
\text { Scheduled to } \\
\text { undergo clinically } \\
\text { indicated invasive } \\
\text { FFR } \\
\text { Willing and able } \\
\text { to give informed } \\
\text { consent }\end{array}$ & $\begin{array}{l}\text { Subjects who because of age, } \\
\text { general medical or psychiatric } \\
\text { condition or physiologic status } \\
\text { cannot give valid informed } \\
\text { consent or tolerate the } \\
\text { coronary CTA examination } \\
\text { Subjects with (severe) renal } \\
\text { insufficiency, indicated as } \\
\text { glomerular filtration rate } \\
\text { (GFR) }<0 \text { mL/min } \\
\text { Subjects with unknown GFR or } \\
\text { obtained }>3 \text { months before the } \\
\text { planned scan } \\
\text { Contraindication or allergy to } \\
\text { intravenous iodinated contrast } \\
\text { agent } \\
\text { Subjects who participate in } \\
\text { another study with radiation } \\
\text { which is estimated to be in risk } \\
\text { category III (ICRP 103) } \\
\text { Subjects who are pregnant } \\
\text { Subjects with contraindications } \\
\text { to cardiac CT and/ } \\
\text { or intravenous contrast, } \\
\text { intravenous adenosine, beta- } \\
\text { blockers or nitroglycerine }\end{array}$ \\
\hline
\end{tabular}

CTA, CT angiography; FFR, fractional flow reserve; ICRP, international commission on radiological protection.

identification of functionally significant coronary artery stenosis. In the targeted population, the sensitivity and specificity of CCTA are currently around $95 \%$ and $50 \%$, respectively. ${ }^{1-3}$ Using SDCT (including CTP), we aim to increase the specificity from $50 \%$ to $85 \% .{ }^{22-24}$ Raising the lower limit of the $95 \%$ CI for specificity from a typical value of $35 \%$ to $60 \%$, necessitates inclusion of at least 66 patients (33 cases and 33 controls). ${ }^{25}$ However, this should not come at a too high cost in sensitivity. Using SDCT (including CTP), we expect a sensitivity around $90 \%$ with a lower limit of the $95 \%$ CI of $65 \%,{ }^{22-24}$ which necessitates inclusion of at least 62 patients (31 cases and 31 controls). ${ }^{25}$ Because we are interested in both the sensitivity and specificity, we take the largest number of patients of the two calculated sample sizes $(n=66)$. To account for attrition, 75 patients will be included. Our internal research also showed that in most cases FFR is measured in more than one vessel, with an average of 1.6 vessels per patient. Thus, for evaluation on a vessel basis, the amount of measurements is expected to be around 120 individual measurements.

\section{Patient recruitment and evaluation}

On fulfilment of the eligibility criteria, patients are asked for participation in the study by their treating physician. After signing informed consent, each study participant will undergo a dedicated CT protocol on an SDCT system before undergoing the clinically indicated invasive FFR measurement. Patients are asked to refrain from coffee consumption from 24 hours prior to imaging. For each patient, a case report form will be filled in, including information from the SDCT scans, invasive FFR measurement(s) and baseline characteristics such as age, gender, medical history, cardiovascular risk factors and demographic information.

\section{SDCT protocol}

All CT acquisitions will be performed using a 64-slice SDCT scanner (IQ on Spectral CT, Philips Healthcare, Best, The Netherlands) with $64 \times 0.625 \mathrm{~mm}$ collimation. All patients will undergo coronary calcium scoring (CCS), adenosine-induced static stress CTP scan and rest CCTA scan. In case of a history of myocardial infarction, an additional late phase acquisition will be performed for myocardial scar detection. An overview of the scan protocol is shown in figure 1. All acquisitions will be performed according to dedicated guidelines. ${ }^{26}$ As contrast agent, $300 \mathrm{mg} \mathrm{I} /$ $\mathrm{mL}$ Iopromide (Ultravist 300, Bayer Healthcare, Berlin, Germany) will be used. For all scans, both conventional and spectral-based images will be reconstructed, which allows accessing DECT options. All images will be analysed using a dedicated workstation (IntelliSpace Portal, Philips Healthcare, Best, The Netherlands).

\section{Coronary calcium scoring}

Acquisitions will be made using a prospectively ECG-triggered (78\% R-R interval) protocol ('Step \& Shoot Cardiac') at $120 \mathrm{kVp}$ and $40 \mathrm{mAs}$. Images will be reconstructed with $3 \mathrm{~mm}$ slice thickness and $3 \mathrm{~mm}$ increment using iterative reconstruction. Coronary calcifications will be quantified with Agatston scores using commercially available software (HeartBeat CS, IntelliSpace Portal, Philips Healthcare, Best, The Netherlands).

\section{Static stress CTP}

On completion of CCS, stress will be induced using intravenous adenosine injection $(140 \mu \mathrm{g} / \mathrm{kg} / \mathrm{min})$. After $3 \mathrm{~min}$ of adenosine injection, a test bolus with $10 \mathrm{~mL}$ iodinated contrast will be given and flushed with $20 \mathrm{~mL}$ saline at a flow rate of $4.5 \mathrm{~mL} / \mathrm{s}(<80 \mathrm{~kg})$ or $5 \mathrm{~mL} / \mathrm{s}(\geq 80 \mathrm{~kg})$. From this test bolus, a patient-specific optimal scan delay is obtained by placing a region of interest in the ascending aorta to determine the time to peak, after which an additional $4 \mathrm{~s}$ are added. Subsequently, the contrast agent will be administered using $71 \mathrm{~mL}$ at $4.5 \mathrm{~mL} / \mathrm{s}(<80 \mathrm{~kg})$ or $85 \mathrm{~mL}$ at $5 \mathrm{~mL} / \mathrm{s}(\geq 80 \mathrm{~kg})$ and flushed with $50 \mathrm{~mL}$ saline at the same flow rate. After the predefined patient-specific optimal scan delay, and still under peak adenosine stress, a retrospectively ECG-gated single CTP scan is acquired in helical mode. The scan is obtained using $120 \mathrm{kVp}$ with cardiac dose modulation for the mAs (reference 270 $\mathrm{mAs})$, with highest $\mathrm{mAs}$ at end-systolic phase (45\% R-R interval) and reduced mAs during the other phases. Directly after the acquisition, the adenosine injection will be stopped. Images will be reconstructed in steps of 

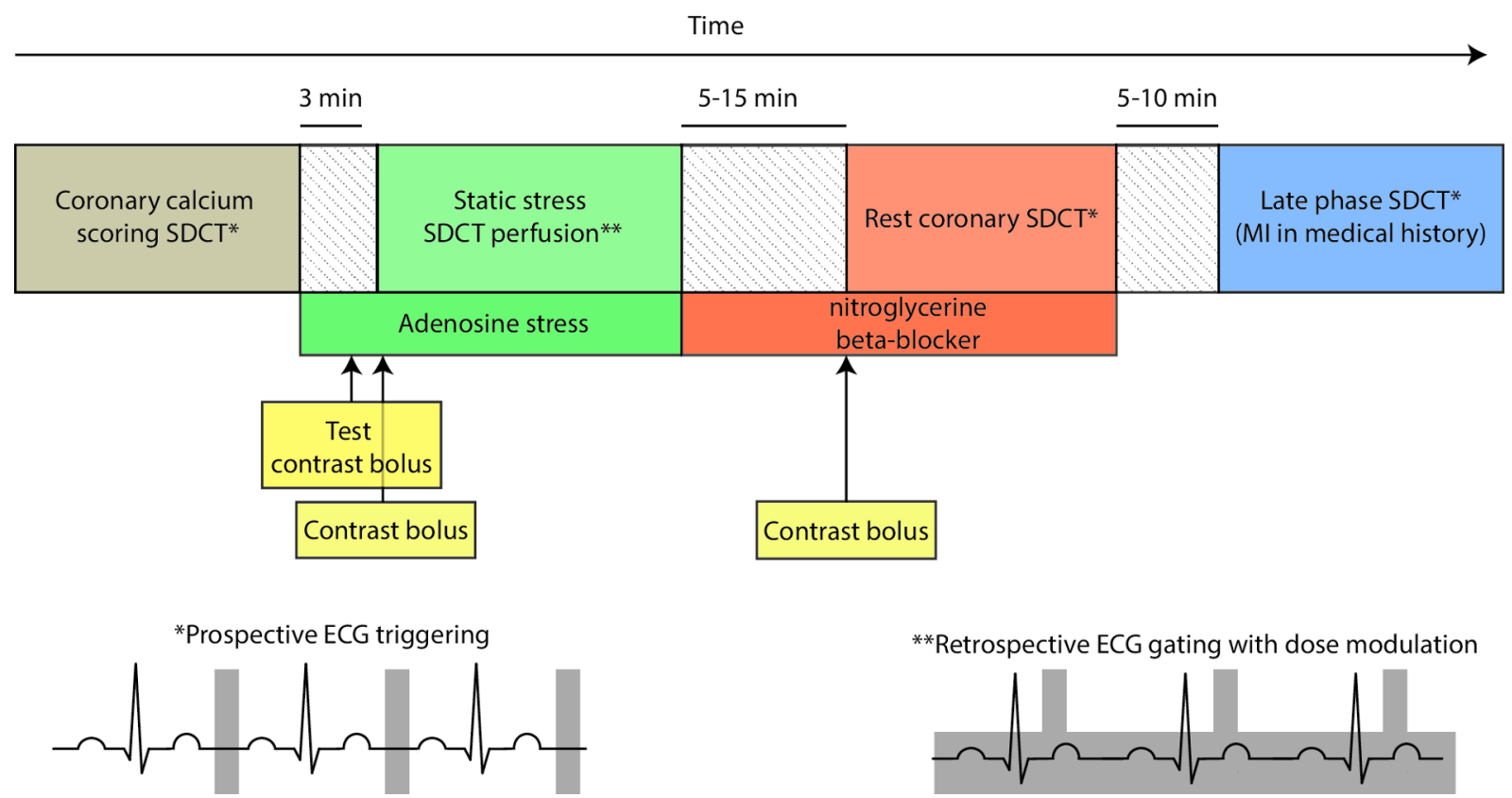

Figure 1 SDCT scan protocol. MI, myocardial infarction; SDCT, dual-layer spectral detector CT.

$10 \%$ from $0 \%$ to $90 \%$ of the R-R interval with $0.9 \mathrm{~mm}$ slice thickness and $0.45 \mathrm{~mm}$ increment using iterative reconstruction. The myocardium will be evaluated using $5 \mathrm{~mm}$ multiplanar reformatted images in short axis and long axis view. Myocardial perfusion will be scored visually and quantitatively on conventional images, monoenergetic images and iodine density images.

\section{Coronary CT angiography}

After a pause of $5-15 \mathrm{~min}$ a resting CCTA will be performed. In accordance with the Society of Cardiovascular CT (SCCT) guidelines, ${ }^{26}$ intravenous beta-blocker will be administered in patients with a heart rate above 60 beats per minute. In all patients, nitroglycerin $0.4 \mathrm{mg}$ is administered sublingually. Iodinated contrast will be administered using the same amount and flow rate as for the stress scan. Seven seconds after a threshold of 100 Hounsfield Units is reached in the descending aorta, a CCTA scan is acquired using a prospectively ECG-triggered (78\% R-R interval) protocol ('Step \& Shoot Cardiac') at $120 \mathrm{kVp}$ and $120 \mathrm{mAs}$. Images will be reconstructed with $0.9 \mathrm{~mm}$ slice thickness and $0.45 \mathrm{~mm}$ increment using iterative reconstruction. In accordance with SCCT guidelines, ${ }^{27} 28$ the images (both conventional and monoenergetic) will be reported and assessed on image quality, plaque characteristics and degree of stenosis (categorised as $0 \%, 1 \%-24 \%, 25 \%-49 \%, 50 \%-69 \%$, $70 \%-99 \%$ and $100 \%)$. For anatomical evaluation of stenosis, $\geq 50 \%$ degree of stenosis will be used to indicate a significant stenosis. In addition to standard assessment, myocardial perfusion will be evaluated and compared with the static stress CTP.

\section{Late phase non-contrast scan}

In case a patient has a history of myocardial infarction an additional late phase non-contrast scan is made $5-10 \mathrm{~min}$ after the rest CCTA scan. This scan is acquired using a prospectively ECG-triggered (78\% R-R interval) protocol ('Step \& Shoot Cardiac') at $120 \mathrm{kVp}$ and $100 \mathrm{mAs}$. Images will be reconstructed with $0.9 \mathrm{~mm}$ slice thickness and $0.45 \mathrm{~mm}$ increment using iterative reconstruction. The myocardium will be evaluated for late enhancement, both visually and quantitatively, using $5 \mathrm{~mm}$ multiplanar reformatted images in short axis and long axis view of the conventional, monoenergetic and iodine density images.

\section{Radiation dose estimation}

The estimated radiation dose for the CT scans will be calculated by multiplying the dose length product with the conversion coefficient for the chest at $120 \mathrm{kVp}$ $(\mathrm{k}=0.0145 \mathrm{mSv} /(\mathrm{mGyxcm})) .^{29}$ The estimated radiation doses for CCS scan, static stress CTP, CCTA and late phase scan are $0.9,2.5-4.4,2.5$ and $2.2 \mathrm{mSv}$, respectively (table 2). Cumulative radiation dose for the total SDCT protocol is estimated to be $\leq 10 \mathrm{mSv}$.

\section{ICA and invasive FFR}

For the clinical purpose, ICA with FFR measurement(s) will be performed in each patient within 30 days after the SDCT scan. Invasive FFR will be measured, under maximal hyperaemia (intravenous adenosine injection $(140 \mu \mathrm{g}$ / $\mathrm{kg} / \mathrm{min}$ )), by placing a pressure wire (PressureWire X Guidewire, St. Jude Medical, St. Paul, Minnesota, USA) distal to the stenosis. The FFR value will be calculated automatically by dividing the pressure measured distally from the stenosis using the pressure wire by the pressure proximally from the stenosis assessed with the guiding catheter. To allow for comparison with $\mathrm{FFR}_{\mathrm{CT}}$, the location of the invasive FFR measurement will be recorded. An FFR value of $\leq 0.80$ will be used as reference standard to indicate a functionally significant stenosis. The FFR value will be blinded for the observers who will perform 
Table 2 Radiation dose estimation

\begin{tabular}{llll}
\hline Type of scan & CTDlvol & DLP* $^{*}$ & $\begin{array}{l}\text { Estimated } \\
\text { radiation dose }\end{array}$ \\
\hline $\begin{array}{l}\text { Coronary calcium } \\
\text { scoring }\end{array}$ & 5 & 63 & $0.9 \mathrm{mSv}$ \\
\hline $\begin{array}{l}\text { Static stress CTP scan } \\
\text { Coronary CTA }\end{array}$ & $14-24 \ddagger$ & $175-300 \ddagger$ & $2.5-4.4 \mathrm{mSv} \ddagger$ \\
\hline $\begin{array}{l}\text { Late phase non- } \\
\text { contrast§ }\end{array}$ & 14 & 175 & $2.5 \mathrm{mSv}$ \\
\hline \begin{tabular}{l} 
Total \\
\hline
\end{tabular} & & 150 & $2.2 \mathrm{mSv}$ \\
\hline
\end{tabular}

${ }^{*}$ Calculated by CTDIvolx $12.5 \mathrm{~cm}$.

†ICRP103 is used to calculate estimated radiation dose whereby the conversion coefficient of 0.0145 is multiplied by the DLP. ${ }^{29}$ ‡Depending on weight.

§Performed in case of a history of myocardial infarction.

CTA, CT angiography; CTP, CT perfusion; CTDIvol, volume CT dose index; DLP dose length product; ICRP, international commission on radiological protection.

CT or FFR $_{\text {CT }}$ readings. And the outcome of the SDCT and image analyses will be blinded for the observer who performs the invasive FFR.

\section{FFR derived from CCTA images}

FFR $_{\text {CT }}$ analysis will be performed on resting CCTA images using a research prototype on-site $\mathrm{FFR}_{\mathrm{CT}}$ method (FFR $\mathrm{FT}_{\mathrm{CT}}$, Intellispace, Philips Healthcare). Coronary artery centreline and lumen will be automatically segmented and manually corrected by an expert. Subsequently, FFR will be automatically calculated and simulated using these segmentations. FFR $_{\mathrm{CT}}$ will be performed on both conventional and monoenergetic images.

\section{DL analyses}

Conventional and SDCT data generated from the different acquisitions will be used as input for the analysis of cardiac anatomy and function using DL. This analysis will include segmentation of anatomical structures of interest (eg, myocardium, coronary arteries), detection and characterisation of atherosclerotic plaque and perfusion defects and subsequent identification of patients with functionally significant stenosis.

\section{Study endpoints and data analysis}

The primary study endpoint is the diagnostic performance of SDCT (including CTP) for the identification of functionally significant coronary artery stenosis defined as FFR $\leq 0.8$. Anatomical evaluation of coronary stenosis will be performed on conventional CCTA images. These data will then be compared with data obtained from SDCT as described above. The secondary study endpoint is the diagnostic performance of SDCT, CTP, FFR $\mathrm{CT}_{\text {and }}$ DL separately and combined for the identification of functionally significant coronary artery stenosis defined as FFR $\leq 0.8$. Diagnostic performance of anatomical evaluation of coronary stenosis, $\mathrm{FFR}_{\mathrm{CT}}$, $\mathrm{CTP}$ and $\mathrm{DL}$ will be evaluated separately using conventional CT reconstruction. Subsequently, the diagnostic performance of each technology including the use of DECT options provided by SDCT (SDCT options) will be assessed separately. In this way, the results of each technique with and without the use of SDCT options can be evaluated and the added value of SDCT for each technique can be defined. Hereafter, FFR ${ }_{\mathrm{CT}}$, CTP and DL (including SDCT options) will be combined with anatomical information. First, the degree of stenosis will be evaluated. If $\geq 25 \%$ degree of stenosis is present on CCTA (+SDCT options), further testing using either FFR $_{\mathrm{CT}}$ (+SDCT options), CTP (+SDCT options) or DL (+SDCT options) will be used to indicate a functionally significant stenosis (figure 2). The DL analysis will be performed on data generated from the different CT acquisitions. For combinations of acquisitions, the DL algorithm will automatically define to which extent the information of each acquisition is weighted in the model.

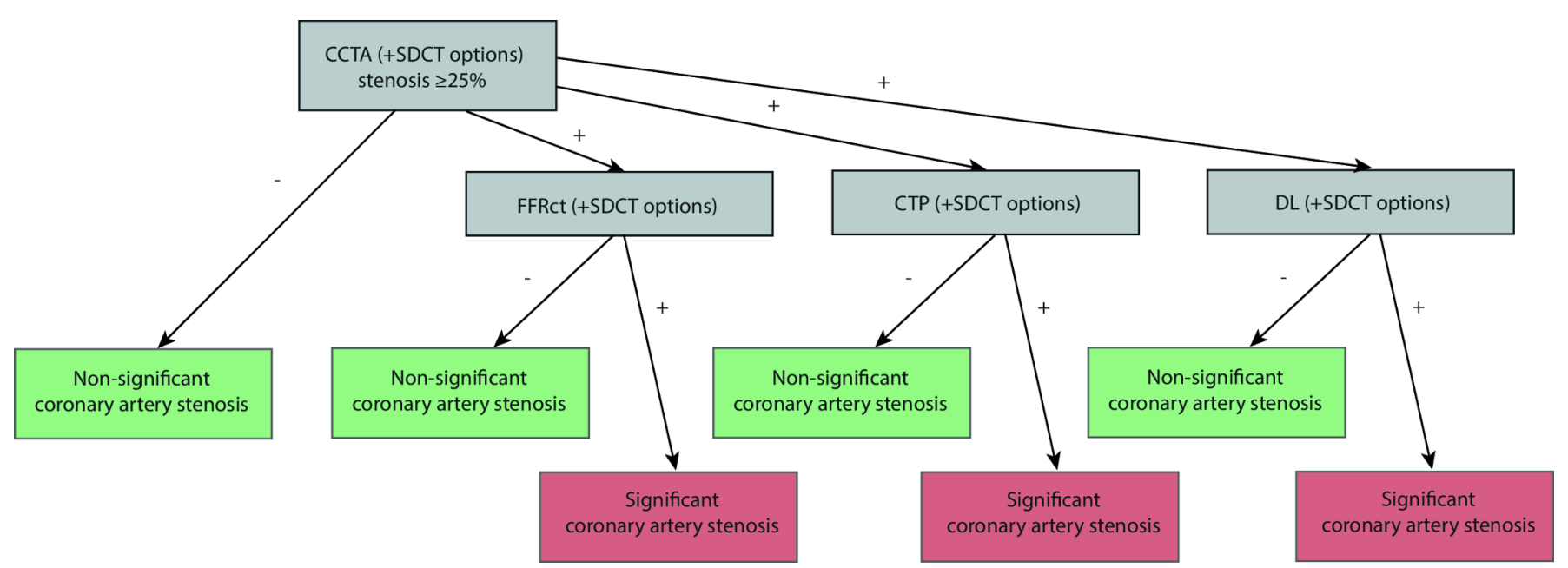

Figure 2 Flow chart of degree of stenosis analysis combined with FFR ${ }_{\mathrm{CT}}$, CTP and DL (including dual-energy CT options provided by SDCT). First, degree of stenosis will be evaluated. If $\geq 25 \%$ degree of stenosis is present, further testing using either FFR ${ }_{\mathrm{CT}}$, CTP or DL will be used to indicate a functionally significant stenosis. CCTA, coronary CT angiography; CTP, CT perfusion; DL, deep learning; $\mathrm{FFR}_{\mathrm{CT}}$, fractional flow reserve derived from coronary CT angiography images; SDCT, dual-layer spectral detector CT. 
For both the primary and secondary objective, the accuracy, sensitivity, specificity, negative predictive value and positive predictive value will be calculated with $95 \%$ CIs on a per-patient level (primary and secondary endpoint) and per-vessel level (secondary endpoint). Differences in diagnostic performance will be investigated using pairwise McNemar tests and area under the receiver-operating curve analyses. A $\mathrm{p}<0.05$ will be considered statistically significant.

The tertiary endpoint is an evaluation of blooming and beam-hardening artefacts of calcifications using SDCT compared with conventional CCTA. To determine the optimal virtual monoenergetic level at which calcium blooming and beam hardening is minimised and anatomy of the vessel is best visualised, degree of stenosis will be quantitatively scored at monoenergetic levels in steps of $10(40-200 \mathrm{keV})$ and on conventional CCTA and compared with degree of stenosis at quantitative coronary angiography (QCA). The monoenergetic image with the least difference in the degree of stenosis compared with QCA is considered best. To determine the difference in blooming and beam-hardening artefacts of calcifications, volumes of circumscribed calcifications will be measured with dedicated volume analysis software (HeartBeat CS, IntelliSpace, Philips Healthcare). The percentage of volume measured on conventional CT minus the volume measured on the monoenergetic level with the least blooming (defined by the quantitative comparison with QCA) is considered as the degree of difference of blooming of calcifications. A paired t-test (normally distributed data) or Wilcoxon signed-rank test (non-normally distributed data) is used to assess the significance of the degree of decrease in blooming. Categorical variables will be expressed as counts and percentages. The Shapiro-Wilk test will be used to identify normally distributed data. Continuous variables will be expressed as mean $\pm \mathrm{SD}$ for normally distributed data and as median with IQR for non-parametric data.

\section{DISCUSSION}

The CLARITY study aims to evaluate the diagnostic performance of (combinations of) new CT acquisition techniques (SDCT and CTP) and image analysis techniques $\left(\mathrm{FFR}_{\mathrm{CT}}\right.$ and $\mathrm{DL}$ ) for the identification of functionally significant coronary artery stenosis. By analysing both anatomical and functional information, we hypothesise that the diagnostic performance of CT can be substantially improved. Multiple studies have demonstrated that assessment with $\mathrm{FFR}_{\mathrm{CT}}{ }^{5}{ }^{6} \mathrm{CTP}^{7-10}$ and image analysis exploiting $\mathrm{DL}^{11} 12$ individually lead to improved diagnostic performance. By combining these techniques with the assessment on monoenergetic and iodine density images, SDCT may allow for improved anatomical and functional evaluation. Each CT acquisition and image analysis technique has its own strengths and weaknesses. FFR $_{\mathrm{CT}}$ can be performed on rest CCTA and thus does not require an additional scan. However, it can be challenging or even impossible in cases with high-density calcified plaque, a stent in the vessel of interest or in the presence of motion or misalignment artefacts. ${ }^{56}$ With the use of SDCT, some of these challenges may be overcome by reduction of blooming and beam-hardening artefacts. ${ }^{15-17}$ Stress CTP on SDCT is less or not influenced by these factors and may potentially be more representative of perfusion defects, as it is acquired during stress. ${ }^{9}$ On the other hand, stress CTP requires an additional scan and administration of adenosine, which increases radiation dose, risk and discomfort, which may be omitted if diagnostic performance is not superior to other techniques. DL-based image analysis may be able to gain insights into optimal combinations of information obtained from the different acquisitions. However, the number of patients in the current study is relatively low for DL-based analysis, which may need to be addressed by designing new approaches for sparsely sampled data. We expect that the highest diagnostic value can be found in a combination of diagnostic tests. The CLARITY study will be the first to investigate the combinations of SDCT, CTP, FFR $\mathrm{CT}_{\text {T }}$ and DL-based image analysis for the identification of functionally significant stenosis. Because the study is monocentric and only patients scheduled to undergo clinically indicated invasive FFR are included, a potential limitation is selection bias. Therefore, the results of the current study will be applicable to a relatively specific population with stable angina and known coronary artery disease. Future studies will be needed to translate the findings of the CLARITY study to a patient cohort with low to intermediate pretest likelihood for obstructive coronary artery disease, clinically more frequently encountered on CT.

\section{CONCLUSION}

The results of the CLARITY study will clarify to what extent SDCT, including CTP and image analysis techniques can improve the specificity for the identification of functionally significant coronary artery stenosis. This could potentially help with clinical decision-making and thereby decrease the number of patients unnecessary referred for invasive FFR.

\section{Status of study}

Patient enrolment started on 29 March 2017 and is anticipated to be completed before 1 August 2020. Until this moment, 21 patients are included in the study.

\section{Ethics and dissemination}

All subjects will provide written informed consent. Study findings will be disseminated through peer-reviewed conference presentations and journal publications.

Contributors Design of the study: RWvH, II, PAdJ, MJW, MV and TL. Principal investigator: TL. Recruitment of patients: RWvH, MJMC, GEHL, MV and TL. Drafted study protocol and manuscript critically for important intellectual content: RWvH. Data collection, analysis and interpretation of the data: all authors. Revised study protocol and manuscript critically for important intellectual content: all authors. All authors have approved the final version of the manuscript. 
Funding This work is funded by the Netherlands Organisation for Scientific Research (NWO) Domain Applied and Engineering Sciences (AES) under project number P15-26. The department of Radiology at University Medical Centre Utrecht receives research support from Philips Healthcare.

Disclaimer The authors are solely responsible for the design and conduct of the study, collection and analysis of the data, writing of the manuscript and its final contents.

Competing interests II received Research grants from Netherlands Organisation for Scientific Research (NWO)/ Foundation for Technological Sciences (number 12726) with industrial participation (Pie Medical Imaging, 3Mensio Medical Imaging). II and TL received research grants from The Netherlands Organisation for Health Research and Development (FSCAD, number 104003009); Research grants from Netherlands Organisation for Scientific Research (NWO) Domain Applied and Engineering Sciences (AES) (number P15-26) with industrial participation (Pie Medical Imaging, Philips Healthcare); Research grants Pie Medical Imaging. MJW and TL gave lectures for the Philips Healthcare Speakers Bureau.

Patient consent for publication Not required.

Ethics approval The study protocol was reviewed and approved by the institutional review board of the University Medical Centre Utrecht (NL55917.041.16). In addition, the radiation safety committee approved this study.

Provenance and peer review Not commissioned; externally peer reviewed.

Open access This is an open access article distributed in accordance with the Creative Commons Attribution Non Commercial (CC BY-NC 4.0) license, which permits others to distribute, remix, adapt, build upon this work non-commercially, and license their derivative works on different terms, provided the original work is properly cited, appropriate credit is given, any changes made indicated, and the use is non-commercial. See: http://creativecommons.org/licenses/by-nc/4.0/.

\section{REFERENCES}

1. Ko BS, Wong DT, Cameron JD, et al. 320-row CT coronary angiography predicts freedom from revascularisation and acts as a gatekeeper to defer invasive angiography in stable coronary artery disease: a fractional flow reserve-correlated study. Eur Radiol 2014;24:738-47.

2. Meijboom WB, Van Mieghem CA, van Pelt N, et al. Comprehensive assessment of coronary artery stenoses: computed tomography coronary angiography versus conventional coronary angiography and correlation with fractional flow reserve in patients with stable angina. J Am Coll Cardiol 2008;52:636-43.

3. Rossi A, Papadopoulou SL, Pugliese F, et al. Quantitative computed tomographic coronary angiography: does it predict functionally significant coronary stenoses? Circ Cardiovasc Imaging 2014;7:43-51.

4. Tonino PA, De Bruyne B, Pijls NH, et al. Fractional flow reserve versus angiography for guiding percutaneous coronary intervention. N Engl J Med 2009;360:213-24.

5. Tesche C, De Cecco CN, Albrecht MH, et al. Coronary CT angiography-derived fractional flow reserve. Radiology 2017;285:17-33.

6. Donnelly PM, Kolossváry M, Karády J, et al. Experience with an onsite coronary computed tomography-derived fractional flow reserve algorithm for the assessment of intermediate coronary stenoses. Am J Cardiol 2018;121:9-13.

7. Rossi A, Merkus D, Klotz E, et al. Stress myocardial perfusion: imaging with multidetector CT. Radiology 2014;270:25-46.

8. Danad I, Szymonifka J, Schulman-Marcus J, et al. Static and dynamic assessment of myocardial perfusion by computed tomography. Eur Heart J Cardiovasc Imaging 2016;17:836-44.

9. Jin KN, De Cecco CN, Caruso D, et al. Myocardial perfusion imaging with dual energy CT. Eur J Radiol 2016;85:1914-21.

10. Meinel FG, De Cecco CN, Schoepf UJ, et al. First-arterial-pass dual-energy CT for assessment of myocardial blood supply: do we need rest, stress, and delayed acquisition? Comparison with SPECT. Radiology 2014;270:708-16.
11. Zreik M, Lessmann N, van Hamersvelt RW, et al. Deep learning analysis of the myocardium in coronary CT angiography for identification of patients with functionally significant coronary artery stenosis. Med Image Anal 2018;44:72-85.

12. van Hamersvelt RW, Zreik M, Voskuil M, et al. Deep learning analysis of left ventricular myocardium in CT angiographic intermediatedegree coronary stenosis improves the diagnostic accuracy for identification of functionally significant stenosis. Eur Radiol 2018.

13. Johnson TR, Fink C, Schönberg SO, et al. Dual energy CT in clinical practice, medical radiology. Berlin, Heidelberg: Springer Berlin Heidelberg, 2011. ISBN 9783642017407.

14. van Hamersvelt RW, Willemink MJ, de Jong PA, et al. Feasibility and accuracy of dual-layer spectral detector computed tomography for quantification of gadolinium: a phantom study. Eur Radiol 2017;27:3677-86.

15. Hickethier T, Baeßler B, Kroeger JR, et al. Monoenergetic reconstructions for imaging of coronary artery stents using spectral detector CT: In-vitro experience and comparison to conventional images. J Cardiovasc Comput Tomogr 2017;11:33-9.

16. Boll DT, Merkle EM, Paulson EK, et al. Calcified vascular plaque specimens: assessment with cardiac dual-energy multidetector CT in anthropomorphically moving heart phantom. Radiology 2008;249:119-26.

17. Symons R, Choi Y, Cork TE, et al. Optimized energy of spectral coronary CT angiography for coronary plaque detection and quantification. J Cardiovasc Comput Tomogr 2018;12.

18. Pelgrim GJ, van Hamersvelt RW, Willemink MJ, et al. Accuracy of iodine quantification using dual energy CT in latest generation dual source and dual layer CT. Eur Radiol 2017;27:3904-12.

19. Litjens $\mathrm{G}$, Kooi T, Bejnordi BE, et al. A survey on deep learning in medical image analysis. Med Image Anal 2017;42:60-88.

20. Wolterink JM, Leiner T, de Vos BD, et al. Automatic coronary artery calcium scoring in cardiac CT angiography using paired convolutional neural networks. Med Image Anal 2016;34:123-36.

21. Gilles M, Sechtem U. Task Force Members. 2013 ESC guidelines on the management of stable coronary artery disease. Eur Heart $J$ 2013;34:2949-3003.

22. Ko SM, Park JH, Hwang HK, et al. Direct comparison of stress- and rest-dual-energy computed tomography for detection of myocardial perfusion defect. Int J Cardiovasc Imaging 2014;30:41-53.

23. Ko SM, Choi JW, Hwang HK, et al. Diagnostic performance of combined noninvasive anatomic and functional assessment with dual-source CT and adenosine-induced stress dual-energy CT for detection of significant coronary stenosis. AJR Am J Roentgenol 2012;198:512-20.

24. Ko BS, Cameron JD, Meredith IT, et al. Computed tomography stress myocardial perfusion imaging in patients considered for revascularization: a comparison with fractional flow reserve. Eur Heart J 2012;33:67-77.

25. Flahault A, Cadilhac M, Thomas G. Sample size calculation should be performed for design accuracy in diagnostic test studies. J Clin Epidemiol 2005;58:859-62.

26. Abbara S, Blanke P, Maroules CD, et al. SCCT guidelines for the performance and acquisition of coronary computed tomographic angiography: a report of the society of cardiovascular computed tomography guidelines committee: Endorsed by the North American Society for Cardiovascular Imaging (NASCI). J Cardiovasc Comput Tomogr 2016;10:435-49.

27. Leipsic J, Abbara S, Achenbach S, et al. SCCT guidelines for the interpretation and reporting of coronary CT angiography: a report of the society of cardiovascular computed tomography guidelines committee. J Cardiovasc Comput Tomogr 2014;8:342-58.

28. Cury RC, Abbara S, Achenbach S, et al. CAD-RADS(TM) Coronary artery disease - reporting and data system. An expert consensus document of the Society of Cardiovascular Computed Tomography (SCCT), the American College of Radiology (ACR) and the North American Society for Cardiovascular Imaging (NASCl). Endorsed by the American College of Cardiology. J Cardiovasc Comput Tomogr 2016;10:269-81.

29. Deak PD, Smal Y, Kalender WA. Multisection CT protocols: sex- and age-specific conversion factors used to determine effective dose from dose-length product. Radiology 2010;257:158-66. 